\title{
Author index to volume 3 (1999)
}

Bakhtazad, A., A. Palazoglu and J.A. Romagnoli, Process data de-noising using wavelet transform

Barrios Bravo, L.J., see del Castillo Sobrino, $\mathbf{M}^{\mathrm{a}}$.D.

Bartsch, H., see Silipo, R.

Bay, S.D., Nearest neighbor classification from multiple feature subsets

$399-408$

$287-306$

191-209

Berry, M.W., see Jiang, J.

Berthold, M.R. and K.-P. Huber, Constructing fuzzy graphs from examples

$377-398$

$37-53$

Black, M. and R.J. Hickey, Maintaining the performance of a learned classifier under concept drift

453-474

Brazdil, P., see Gama, J.

$1-22$

Bui, H.H., S. Venkatesh and G. West, Layered dynamic probabilistic networks for spatio-temporal modelling

$339-361$

Cardoso, M.G.M.S., I.H. Themido and F.M. Pires, Evaluating a clustering solution: An application in the tourism market

Chi, S.-C., see Hong, T.-P.

Chien, S., F. Fisher, E. Lo, H. Mortensen and R. Greeley, Using artificial intelligence planning to automate science image data analysis

Cook, D.J., see Su, S.

Davidsson, P., Integrating models of discrimination and characterization

de Sousa, M.S.R., M. Mattoso and N.F.F. Ebecken, Mining a large database with a parallel database server

Deco, G., see Silipo, R.

del Castillo Sobrino, $\mathbf{M}^{\text {a }}$.D. and L.J. Barrios Bravo, Knowledge acquisition from batch semiconductor manufacturing data

Donato, J.M., see Jiang, J.

Ebecken, N.F.F., see de Sousa, M.S.R.

Fisher, F., see Chien, S.

Frieß, T-T. and R.F. Harrison, A kernel-based Adaline for function approximation

Fu, Z., Dimensionality optimization by heuristic greedy learning vs. genetic algorithms in knowledge discovery and data mining

Gama, J. and P. Brazdil, Linear tree

Golob, M., Decomposition of a fuzzy controller based on the inference break-up method

Grady, N.W., see Jiang, J.

Greeley, R., see Chien, S.

Harrison, R.F., see Frieß, T-T.

Hickey, R.J., see Black, M.

Hickey, R.J., see Hunniford, T.J.C.

Holder, L.B., see $\mathrm{Su}$, S.

$413-436$

Honavar, V., see Yang, J.

Hong, T.-P. and C.-Y. Lee, Effect of merging order on performance of fuzzy induction

$139-151$

Hong, T.-P., C.-S. Kuo and S.-C. Chi, Mining association rules from quantitative data

Huber, K.-P., see Berthold, M.R. 
Hunniford, T.J.C. and R.J. Hickey, A simulated annealing technique for generating artificial data to assess concept learning algorithms

Jappy, P., see Nock, R.

Jiang, J., M.W. Berry, J.M. Donato, G. Ostrouchov and N.W. Grady, Mining consumer product data via latent semantic indexing

Kalousis, A. and T. Theoharis, NOEMON: Design, implementation and performance results of an intelligent assistant for classifier selection

Kuo, C.-S., see Hong, T.-P.

Lee, C.-Y., see Hong, T.-P.

Li, H.L. and J.R. Yu, A piecewise regression analysis with automatic change-point detection

Lo, E., see Chien, $\mathbf{S}$.

Mattoso, M., see de Sousa, M.S.R.

$437-451$

Mortensen, H., see Chien, S.

Nock, R. and P. Jappy, Decision tree based induction of decision lists

Ostrouchov, G., see Jiang, J.

$377-398$

Palazoglu, A., see Bakhtazad, A.

Parekh, R., see Yang, J.

Pfeiffer, D.U., see Stärk, K.D.C.

Pires, F.M., see Cardoso, M.G.M.S.

491-510

Potamias, G., MICSL: Multiple Iterative Constraint Satisfaction based Learning

Romagnoli, J.A., see Bakhtazad, A.

Silipo, R., G. Deco and H. Bartsch, Brain tumor classification based on EEG hidden dynamics

Stärk, K.D.C. and D.U. Pfeiffer, The application of non-parametric techniques to solve classification problems in complex data sets in veterinary epidemiology - An example

Su, S., D.J. Cook and L.B. Holder, Knowledge discovery in molecular biology: Identifying structural regularities in proteins

Themido, I.H., see Cardoso, M.G.M.S.

Yang, J., R. Parekh and V. Honavar, DistAl: An inter-pattern distance-based constructive learning algorithm 